\title{
Toxicometabolomics of lindane in adult zebrafish (Danio rerio) using GC-MS/MS and LC-Orbitrap-MS/MS
}

\author{
Xiu Yuan', Junghak Lee ${ }^{1}$, Eunyoung Park', Hwa-Kyung Lee ${ }^{2^{*}}$ and Jeong-Han Kim ${ }^{1^{*}}$ (D)
}

\begin{abstract}
Lindane is a broad-spectrum persistent organochlorine pesticide that has been used to control pests for many years. In this study, its toxic mechanisms in adult zebrafish were investigated using targeted metabolomics with GC-MS/MS and non-targeted metabolomics with LC-Orbitrap-MS/MS. Zebrafish was exposed to lindane in water for $48 \mathrm{~h}$ in three groups: control, low exposure $\left(1 / 10 \mathrm{LC}_{50}\right)$ and high exposure $\left(\mathrm{LC}_{50}\right)$. In the zebrafish exposed to low concentration of lindane, $2.24-3.98 \mathrm{mg} / \mathrm{kg}$ of lindane were determined, while $35.67-56.46 \mathrm{mg} / \mathrm{kg}$ were observed in the zebrafish exposed to high concentration. A total of 118 metabolites were identified from 394 metabolites on GC-MS/MS and 45 metabolites were selected as biomarkers. A total of 62 metabolites were identified on LC-Orbitrap-MS/MS and 7 metabolites were selected as biomarkers. Three groups were well separated on partial least squares-discriminant analysis (PLS-DA), and a total of 52 metabolites in both the targeted and non-targeted metabolites were selected as biomarkers through VIP and ANOVA tests to construct a heatmap. Five metabolic pathways such as the pentose phosphate pathway (PPP), histidine metabolism, phenylalanine metabolism, alanine/aspartate/glutamate metabolism, and phenylalanine/tyrosine/tryptophan biosynthesis, were observed to show toxicologically significant alterations. Oxidative stress was also confirmed through MDA and ROS assays. Such perturbations of the metabolic pathways of zebrafish caused by the exposure to lindane resulted in significant toxicological effects.
\end{abstract}

Keywords: Metabolomics, Lindane, Zebrafish, GC-MS/MS, LC-Orbitrap-MS/MS

\section{Introduction}

Chemicals called persistent organic pollutants (POPs) can cause environmental problems because they are predominant toxic contaminants with long half-lives to remain in the environment [1]. In particular, organochlorine pesticides POPs have carbon-chlorine bonds, which are very stable to hydrolysis and the many chlorine substituents and/or functional groups make them more resistance to biological degradation and photolysis [2]. It was noted that during the 1950s-1970s that

\footnotetext{
*Correspondence: hklee@yuhan.ac.kr; kjh2404@snu.ac.kr

${ }^{1}$ Department of Agricultural Biotechnology and Research Institute

of Agriculture and Life Sciences, Seoul National University, Seoul 08826, Republic of Korea

2 Department of Yuhan Biotechnology, Yuhan University, Bucheon 14780, Gyeonggi-do, Korea
}

organochlorine pesticides such as lindane and DichloroDiphenyl-Trichloroethane (DDT) accounted for approximately $80 \%$ of total pesticides produced [3]. For this reason, organochlorine pesticides continue to cause environmental problems in many countries [4-6].

Lindane is a POP that is a broad-spectrum persistent organochlorine pesticide and has been used to control pests for many years [7]. It has inhibitory effects on gamma amino butyric acid (GABA) receptors in insects and vertebrates $[8,9]$. The chloride channel blocking mechanism of lindane may involve non-competitive binding to channel-associated allosteric sites on the receptor. Toxic symptoms caused by lindane include spontaneous increases in nerve activity and convulsive seizures in insects and vertebrates [10]. Lindane produced various metabolites in bio-environmental systems 
such as hexachorocyclohexene, pentachlorocyclohexene, tetrachlorobenzenes, trichlorobenzenes, dichlorobenzene, tetrachlorophenol, trichlorophenols, dichlorophenol and dichlorophenylglutathiones and etc. [11]. The US Environmental Protection Agency banned lindane use in August 2006, but the environmental problems associated with lindane remains [12]. In the environment, lindane is more soluble in water than most other organochlorine compounds therefore it has a greater possibility of remaining in the water column [13, 14]. Because of its strong hydrophobic property and long persistency, lindane can bioaccumulate in fish, microorganisms, and mammals $[15,16]$. Therefore, it is necessary to conduct research on lindane exposure in aquatic ecosystems. Zebrafish are known as model organisms for studies of physiology, vertebrate biology, and human disease [17]. In a human drug conversion study, about $86 \%$ of the 1318 human drug targets have been identified in zebrafish [18]. Many previous metabolomics studies have been conducted with zebrafish because of their special feature like the genetic homology with mammals, prolificacy, portable size, and economics [19-23].

From the 1990s, metabolomics was defined as post genomics area and is the closest to the phenotype in the "omics cascade" to be the end point of that cascade. It aimed to identify the changes of biochemical pathways of animals, plants and microbes in response to physiological stimuli or genetic disturbances. Therefore, measuring metabolites present in cells, tissues or organisms is a key task in metabolomics research, however, there is currently no single technological platform to analyze all metabolites [24, 25]. In metabolomics analytical techniques, the most commonly used analytical methods are those using GC-MS, LC-MS, and NMR [26]. Each of these technologies has its own unique advantages and disadvantages. Many previous studies have been conducted to confirm the biological responses to environmental stress using NMR $[21,27,28]$. The main disadvantage of NMR is that it is a relatively low-sensitivity technique that has a high detection limit of about $1-5 \mu \mathrm{M}$ and requires a relatively large sample amount $(\sim 500$ $\mu \mathrm{L})$ [29]. Compared with NMR, MS can separate many metabolites with high resolution and sensitivity [30]. GC-MS-based metabolomics can cover a certain polarity range [31]. In addition, GC-MS is an ideal analytical equipment for the analysis of both volatile and non-volatile compounds through derivatization of non-volatile polar compounds. The latest capillary GC is an excellent tool for the analysis of complex metabolic mixtures, providing the chromatographic separations with high resolution and reproducibility [30]. Moreover, compared to LC-MS based analysis, GC-MS has the advantage of much greater resolution, and has a large spectral library, and can separate small compounds that tend to be eluted to the solvent front early in conventional reverse-phase LC-MS analysis into good retention [32]. Therefore, in targeted metabolomics study, GC-MS has the advantage of being able to accurately and quantitatively detect more diverse metabolites than LC-MS. However, LC-OrbitrapMS-based metabolomics provides positive and negative ionization, which increases the variety of metabolites that can be detected [31,33]. These key properties have led to an increase in the use of Ultra-high performance liquid chromatography tandem mass spectrometry (UHPLCMS/MS) related procedures in recent years [34, 35]. The strategies used for metabolomics experiments include 'targeted' and 'non-targeted' metabolomics [36]. Targeted metabolomics uses multiple analyses of defined metabolites that are characterized chemically and annotated biochemically [24]. On the other hand, non-targeted metabolomics uses a comprehensive method to analyze unknown metabolites [37].

In this study, both GC-MS/MS-based targeted metabolomics and LC-Orbitrap-MS/MS-based non-targeted metabolomics were performed after zebrafish exposure to lindane. A total of 394 metabolomes were analyzed by GC-MS/MS with multiple reaction monitoring (MRM) mode in targeted metabolomics, and LC-Orbitrap-MS/ MS with scan mode was used in non-targeted metabolomics to identify metabolites in zebrafish. Statistical analyses were performed by SIMCA ${ }^{+}$and MetaboAnalyst 5.0 to identify biomarker metabolomes and obtain a heatmap. The metabolic pathways were identified by MetaboAnalyst 5.0 to investigate the toxicological and biochemical effects of lindane in zebrafish.

\section{Materials and methods} Chemicals and reagents

Analytical-standard lindane (98.5\%) was purchased from Dr. Ehrenstorfer GmbH (Augsburg, Germany), and ribitol, as an internal standard, was purchased from Wako. TMCS-MSTFA reagent (1\%) was purchased from Thermo Fisher Scientific Company. Methoxyamine hydrochloride, N,N-dimethylformamide (DMF), and pyridine were purchased from Sigma-Aldrich. Acetonitrile and water were of LC-MS grade and were purchased from Fisher Scientific. Acetone and methanol were purchased from Merck (Darmstadt, Germany) and were of LC-MS grade. Reagents with the highest available grades were used. The methoxyamination reagent was prepared by dissolving $20 \mathrm{mg}$ of methoxyamine hydrochloride in $1 \mathrm{~mL}$ of pyridine.

\section{Experimental animals and chemical exposure}

Chemical exposure were carried out as previously described [38] with slight modification. Adult zebrafish 
with the body weight of 400-500 $\mathrm{mg}$ and the length of $3-4 \mathrm{~cm}$ were used, and gender was not considered. Adult zebrafish were acclimated at $23 \pm 1{ }^{\circ} \mathrm{C}$ in a glass water tank for 1 week. The photoperiod was maintained as a 16:8 h light:dark cycle. During this period, zebrafish were fed commercial fish feed, and were not fed on the day before lindane exposure. The $\mathrm{LC}_{50}$ value of lindane for zebrafish is $110 \mu \mathrm{g} / \mathrm{L}$ for $48 \mathrm{~h}$ [39]. Based on this value, the exposure concentrations were set to $110 \mu \mathrm{g} / \mathrm{L}$ $(1100 \mu \mathrm{g} / \mathrm{mL}$ in acetone, $0.5 \mathrm{~mL})$ and $11 \mu \mathrm{g} / \mathrm{L}(110 \mu \mathrm{g} / \mathrm{mL}$ in acetone, $0.5 \mathrm{~mL}$ ) for the high-exposure and low-exposure groups, respectively.

In $10 \mathrm{~L}$ beakers, 20 zebrafish per group were kept for $48 \mathrm{~h}$ before exposure to $110 \mu \mathrm{g} / \mathrm{L}$ (high exposure; $\mathrm{LC}_{50}$ ) and $11 \mu \mathrm{g} / \mathrm{L}$ (low exposure; $1 / 10 \mathrm{LC}_{50}$ ) of lindane, including a control group without lindane. After exposure, 6 zebrafish in each group were sampled for metabolome and oxidative stress analyses. During the experiment, the exposure solution was changed daily to maintain the desired lindane concentration.

\section{Metabolomic sample preparation, profiling and metabolome identification by GC-MS/MS}

Sample preparation procedure was performed as previously described [38]. Fish samples were frozen in liquid $\mathrm{N}_{2}$ in $5 \mathrm{~mL}$ Eppendorf tubes with 3 of $3 \mathrm{~mm}$ and 2 of $7 \mathrm{~mm}$ Retsch stainless steel were ground by shaking vigorously using a MiniG (SPEX Sample Prep, USA). One $\mathrm{mL}$ of $50 \% \mathrm{MeOH}$ solution containing $0.1 \mu \mathrm{g} /$ $\mathrm{mL}$ of ribitol (internal standard) was added to a $50 \mathrm{mg}$ of ground sample in a $2 \mathrm{~mL}$ tube, and the sample was then vortexed at $4{ }^{\circ} \mathrm{C}$ for $10 \mathrm{~min}$. After centrifuging the extracts for $10 \mathrm{~min}$ at $13,000 \mathrm{rpm}, 100 \mu \mathrm{L}$ of supernatants of the extracts were evaporated by a speed vacuum concentrator (Hanil Modulspin 40). The dried residues were reacted with $50 \mu \mathrm{L}$ of methoxyamination reagent for $90 \mathrm{~min}$ at $37{ }^{\circ} \mathrm{C}$ with shaking and were then derivatized with $50 \mu \mathrm{L}$ of MSTFA reagent (MSTFA $+1 \%$ TMCS) for $30 \mathrm{~min}$ at $37^{\circ} \mathrm{C}$.

For metabolite analysis, a Shimadzu GCMS-TQ8040 equipped with a BPX-5 column $(30 \mathrm{~m} \times 0.25 \mathrm{~mm}$ i.d., $0.25 \mu \mathrm{m}$ film thickness, TRAJAN) was used in MRM mode and a total of 394 metabolites (332 metabolites from the Smart Metabolites Database, and 62 metabolites from an in-house library) were analyzed. The injection volume was $1.0 \mu \mathrm{L}$ (split mode; 30:1), and the temperatures for the injector, transfer lines and ion source $(70 \mathrm{eV})$ were $250{ }^{\circ} \mathrm{C}, 280{ }^{\circ} \mathrm{C}$ and $200{ }^{\circ} \mathrm{C}$, respectively. The initial oven temperature was $60^{\circ} \mathrm{C}$ [2 min], and the temperature was ramped to $320{ }^{\circ} \mathrm{C}$ at a rate of $10{ }^{\circ} \mathrm{C} / \mathrm{min}$ and then maintained for $15 \mathrm{~min}$. Helium was used as the carrier gas with a flow rate of $1 \mathrm{~mL} / \mathrm{min}$ and argon was used as the collision gas. Data processing was performed with manual reconfirmation of the peak detections using GCMS Solution software (version 4.3, Shimadzu).

\section{Non-targeted metabolite profiling and identification by LC-Orbitrap-MS/MS}

Non-targeted metabolite profiling and identification were conducted as previously described [40] with slight modification. Fish extract $(5 \mu \mathrm{L})$ before derivatization from the targeted metabolomics method was used for non-targeted metabolite analysis, which employed a Q-Exactive Orbitrap mass spectrometer (Thermo Fisher Scientific Inc., Waltham, MA) that was coupled with a UHPLC (DGU-20A) system. The separations were performed at $50^{\circ} \mathrm{C}$ with an HSS T3 C18 column $(2.1 \times 100 \mathrm{~mm}, 1.8 \mu \mathrm{m}$, Advanced Materials Technology, Wilmington, DE). The flows were constant at $0.3 \mathrm{~mL} / \mathrm{min}$ with $0.1 \%$ formic acid in water (A) and $0.1 \%$ formic acid in methanol (B) as the mobile phases. The following gradient was used: $10 \% \mathrm{~B}$ for $0-0.2 \mathrm{~min}, 10 \%$ to $55 \%$ for $0.2-15 \mathrm{~min}, 55 \%$ to $98 \%$ for $15-20 \mathrm{~min}, 98 \%$ B for $20-35 \mathrm{~min}, 98 \%$ to $10 \%$ B for 35-35.1 min and 10\% B for 35.1-40 min.

Mass spectra were obtained with an HESI (heated electrospray ionization) source that was operated in positive and negative modes. The parameters for the HESI source were set as follows: the sheath gas flow rate was 35 (arbitrary units), auxiliary gas flow rate was 10 (arbitrary units), spray voltage was $4 \mathrm{kV}$, and heater temperature was $350^{\circ} \mathrm{C}$. The MS was set to full scan with ddMS ${ }^{2}$ from $\mathrm{m} / \mathrm{z} 100$ to 1500 . In the data interpretation process, the mass error range was set to $5 \mathrm{ppm}$ or less, and the metabolites were identified to have a mzCloud matching score of $80 \%$ or more.

\section{Determination of lindane concentration in zebrafish using GC-MS/MS}

Lindane was extracted from $200 \mu \mathrm{L}$ of fish extract before derivatization with $200 \mu \mathrm{L}$ of hexane. One $\mu \mathrm{L}$ of hexane layer was analyzed with GC-MS/MS. Calibration curve solutions $(10,20,50,100$ and $200 \mu \mathrm{g} / \mathrm{L})$ were prepared with control fish extract to compensate matrix effect, and they were also extracted with hexane before GC-MS/MS analysis. Analytical condition of GC-MS/MS was identical with metabolites analysis as described above with specific MRM mode for lindane (quantification: $\mathrm{m} / \mathrm{z}$ $181.0 \mathrm{~m} \rightarrow / z 145.0$, and qualification: $m / z 219.0 \mathrm{~m} \rightarrow / z$ 183.0). Samples exposed to high concentration of lindane were diluted by $10-20$-fold with hexane for quantitation. 


\section{Statistical analysis of metabolomes and metabolic pathway analysis}

Multivariate data analyses were performed using SIMCA-P ${ }^{+}$software (version 12.0.1, Umetrics, Sweden). The supervised multidimensional statistical model, which is a partial least squares-discriminant analysis (PLS-DA), was conducted to differentiate between each treatment group. By confirming the VIP (variable importance in the projection) scores in PLS-DA, metabolites with VIP scores $>1$ and standard errors $<1$ were selected. The $\mathrm{p}$-value of selected metabolites were calculated using one-way ANOVA, and metabolites with $\mathrm{p}<0.05$ were selected as biomarker metabolites affected significantly by lindane exposure. A heatmap of selected biomarker metabolites was generated using MetaboAnalyst 5.0 (www.metaboanalyst.ca). MetaboAnalyst 5.0 also produced the metabolic pathway analysis plots based on the Danio rerio KEGG library.

\section{Measurement of ROS and MDA contents}

ROS and MDA contents were measured according to manuals with commercially available kits, respectively. The contents of reactive oxygen species (ROS) were measured by DCFH-DiOxyQ (OxiSelect ${ }^{\mathrm{TM}}$ In vitro ROS/ RNS assay kit, Cell Biolabs, Inc., CA, USA). After adding $500 \mu \mathrm{L}$ of phosphate buffered saline (PBS) to a $20 \mathrm{mg}$ of powder sample, it was homogenized and centrifuged at $10,000 \mathrm{~g}$ for $5 \mathrm{~min}$. Fifty microliters of the supernatant was mixed with $50 \mu \mathrm{L}$ of catalyst and incubated at room temperature for $5 \mathrm{~min}$. DCFH solution $(100 \mu \mathrm{L})$ was added to the incubated mixture before further incubation for $40 \mathrm{~min}$ at room temperature. Fluorescence intensities of incubated samples were measured using a spectrophotometer (SpectraMax i3, Molecular Devices) at $480 \mathrm{~nm}$ excitation/530 $\mathrm{nm}$ emission.

The contents of malondialdehyde (MDA) were determined using a thiobarbituric acid (TBA) assay (OxiSelect $^{\mathrm{TM}}$ TBARS assay kit, Cell Biolabs, Inc., CA, USA). Into $30 \mathrm{mg}$ of sample powder, $500 \mu \mathrm{L}$ of $1 \mathrm{X}$ butylated hydroxytoluene (BHT) solution was added and it was centrifuged at $10,000 \mathrm{~g}$ for $5 \mathrm{~min}$ after homogenization. One hundred microliters of the supernatant was mixed with $100 \mu \mathrm{L}$ of SDS lysis solution and incubated at room temperature for $5 \mathrm{~min}$. A $250 \mu \mathrm{L}$ of TBA reagent was added to the incubated mixture and further incubated for $60 \mathrm{~min}$ at $95{ }^{\circ} \mathrm{C}$. After the incubation was completed, the samples were cooled to room temperature and were centrifuged at $3000 \mathrm{rpm}$ for $15 \mathrm{~min}$. To prevent interference with hemoglobin and its derivatives, $300 \mu \mathrm{L}$ of the supernatant was extracted with $300 \mu \mathrm{L}$ of butanol. The fluorescence intensities of the samples in the butanol layer were measured using a spectrophotometer (SpectraMax i3, Molecular Devices) at $540 \mathrm{~nm}$ excitation/590 nm emission.

\section{Results and discussion}

Determination of lindane concentrations in zebrafish using GC-MS/MS

Lindane was extracted from fish extract with hexane [41] and analyzed with GC-MS/MS. Retention time of lindane was $16.8 \mathrm{~min}$. Linearity of calibration curve (r2) was $>0.99$, indicating good correlation. In the zebrafish exposed with low concentration of lindane, 2.24-3.98 mg/ $\mathrm{kg}$ of lindane were determined, while $35.67-56.46 \mathrm{mg} / \mathrm{kg}$ were observed in the zebrafish exposed with high concentration (Table 1).

\section{Metabolome profiling using GC-MS/MS and LC-Orbitrap-MS/MS}

Metabolomics analysis was performed using the 'targeted' and 'non-targeted' methods on GC-MS/MS and LCOrbitrap-MS/MS. Recently, many metabolomics studies have been conducted using GC-MS and LC-MS, which are complementary mass spectrometry techniques. These techniques can identify an extensive range of metabolites with good sensitivity and selectivity and can provide complementary data to save experimental time with a single sample preparation process [42-44]. In targeted metabolomics, 394 metabolites (332 metabolites were from the Smart Metabolites Database and 62 metabolites were from our own laboratory library) were analyzed using GC-MS/ MS with MRM mode. These metabolites included 78 carbohydrates, 41 fatty acids, 18 alcohols, 75 amino acids, 9 nucleosides, 102 organic acids, 10 amines, 7 pyridines, 4 indoles, 11 purines, 2 nucleotides, 10 amides, 2 tocopherols, 6 steroids, 9 phenols, 4 pyrimidines, 3 esters, 1 inorganic compound, 2 isoprenoids, 1 glyceride, and 1 azole. After the GC-MS/MS analyses, manual identification of peaks was performed by confirming the corresponding metabolite MRM ions, peak retention times and areas. In these analyses, 118 confirmed metabolites were detected in whole zebrafish bodies (Fig. 1).

In non-targeted metabolomics, a total of 62 metabolites were detected and identified after LC-Orbitrap-MS/MS analysis and data processing with Compound Discoverer 3.1 (CD3.1). These metabolites included the following: 7 amines, 14 amino acids, 7 carbohydrates, 9 fatty acids, 2 fatty acyls, 3 steroids, 3 purines, 7 organic acids, 2 glycerides, 1 alcohol, 1 indole, 1 polyethylene glycol, 1 pterin, 1 pyridine, 1 stilbene, 1 aniline, and 1 organosulfonic acid (Fig. 2).

Table 1 The concentration of lindane in zebrafish

\begin{tabular}{lrrrr}
\hline Sample & $\mathbf{1}$ & $\mathbf{2}$ & $\mathbf{3}$ & \multicolumn{1}{c}{ Average } \\
\hline Low exposure & $2.24 \mathrm{mg} / \mathrm{kg}$ & $3.98 \mathrm{mg} / \mathrm{kg}$ & $3.79 \mathrm{mg} / \mathrm{kg}$ & $3.34 \mathrm{mg} / \mathrm{kg}$ \\
High exposure & $35.67 \mathrm{mg} / \mathrm{kg}$ & $42.32 \mathrm{mg} / \mathrm{kg}$ & $56.46 \mathrm{mg} / \mathrm{kg}$ & $44.81 \mathrm{mg} / \mathrm{kg}$ \\
\hline
\end{tabular}




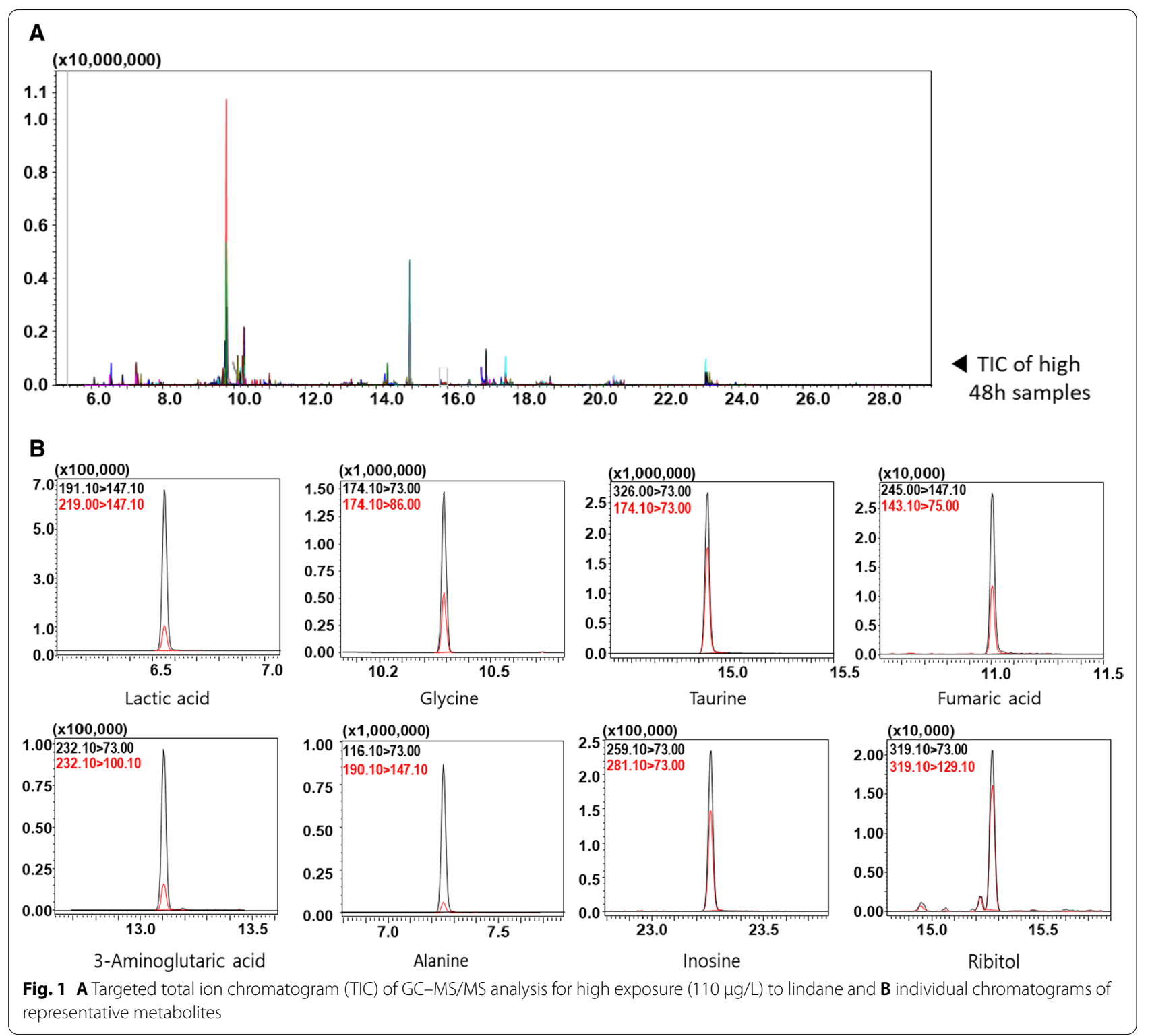

\section{Metabolomic alteration induced by lindane exposure}

According to the PLS-DA plot of the targeted metabolomics data, the control group and 2 treated groups showed significant differences in their metabolic profiles (Fig. 3). VIP analyses of the PLS-DA model and p-value analyses of the ANOVA model were conducted to obtain 45 metabolites as biomarkers that contributed significantly to differentiating the metabolic profiles between the control and the two exposed groups (Table 2). From the same statistical analysis using non-targeted metabolomics data, 7 additional biomarker metabolites were identified. Table 3 shows the list of biomarker metabolites that were finally identified from the non-targeted method, including their molecular formulas, adducts, molecular weights, retention times,
mzCloud matching scores and HMDB numbers. Therefore, a total of 52 biomarkers were found as a result of the statistical analyses of targeted and non-targeted metabolome data. The heatmap of these biomarkers clearly shows the pattern of metabolite changes (Fig. 4). The colors in the heatmap represent the metabolite levels from highest (red) to lowest (blue), which demonstrate a clear difference between the control and treatment groups.

\section{Metabolic pathway and function analysis}

Metabolic pathway analysis was carried out using MetaboAnalyst 5.0 with the Kyoto Encyclopedia of Gene and Genomes (KEGG). Five main perturbed pathways were identified, including the pentose phosphate pathway 

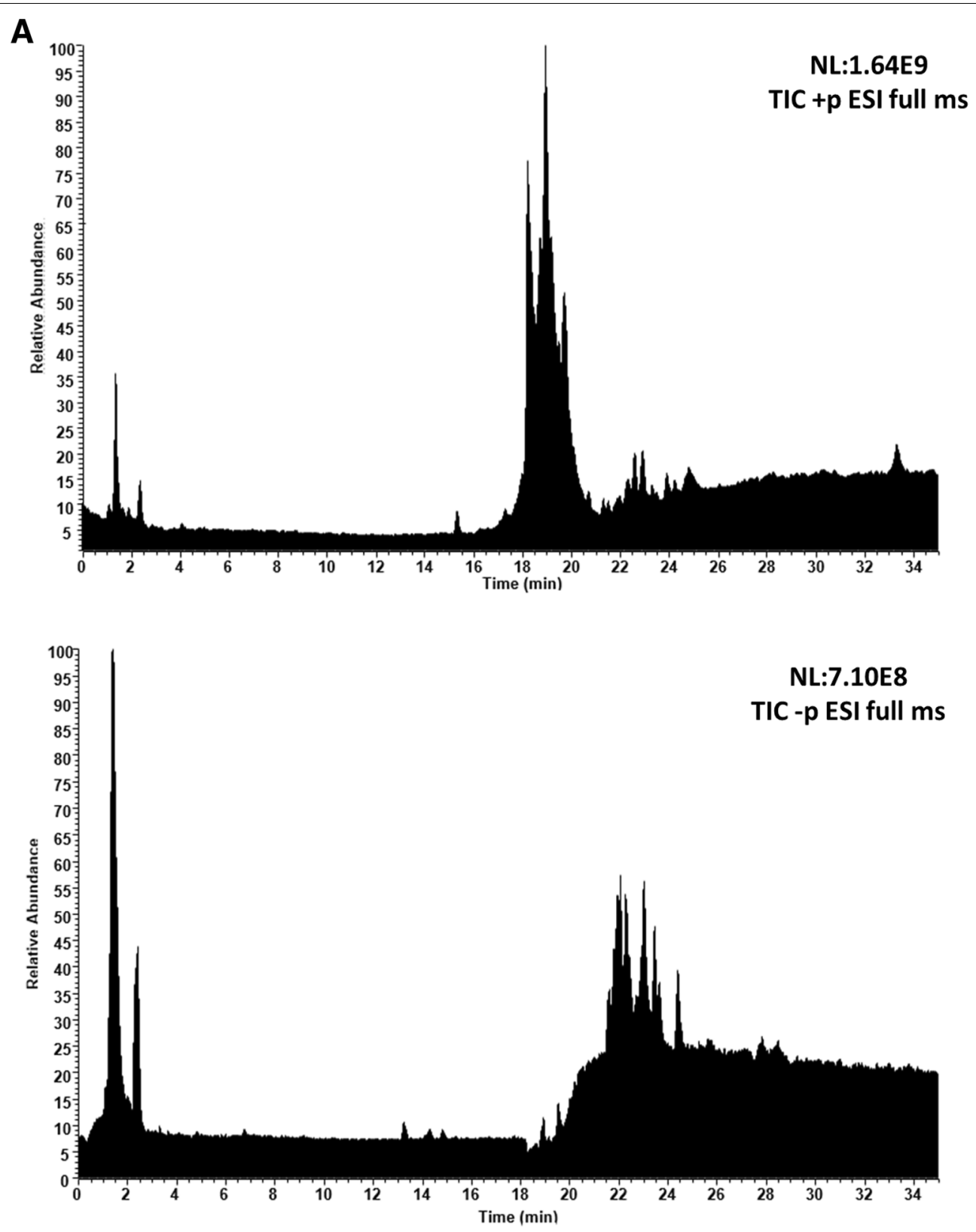

B
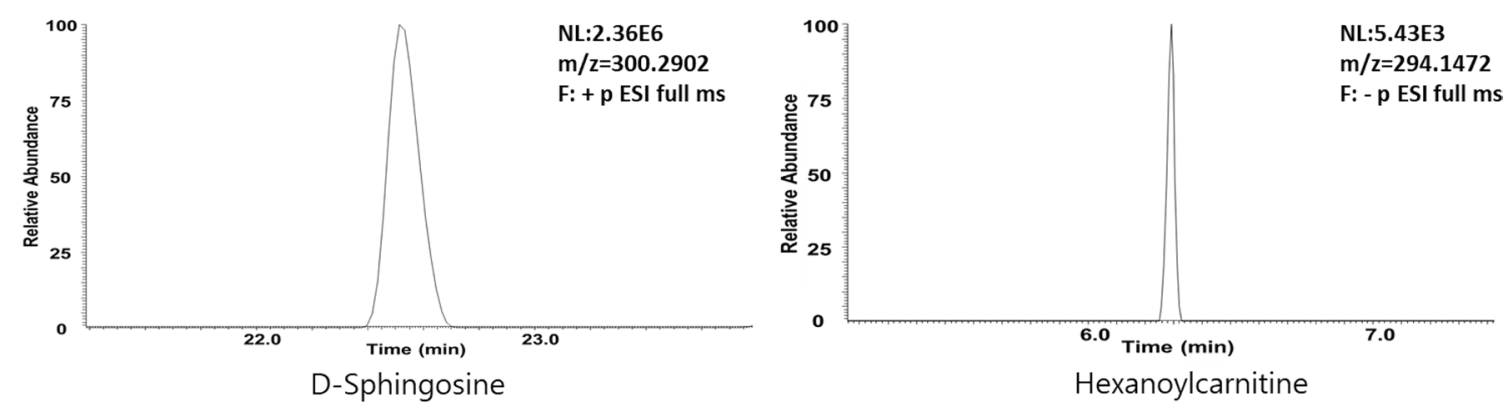

Fig. 2 A Non-targeted total ion chromatogram of LC-Orbitrap-MS analysis for high exposure (110 $\mu \mathrm{g} / \mathrm{L})$ to lindane and $\mathbf{B}$ individual chromatograms of representative metabolites 


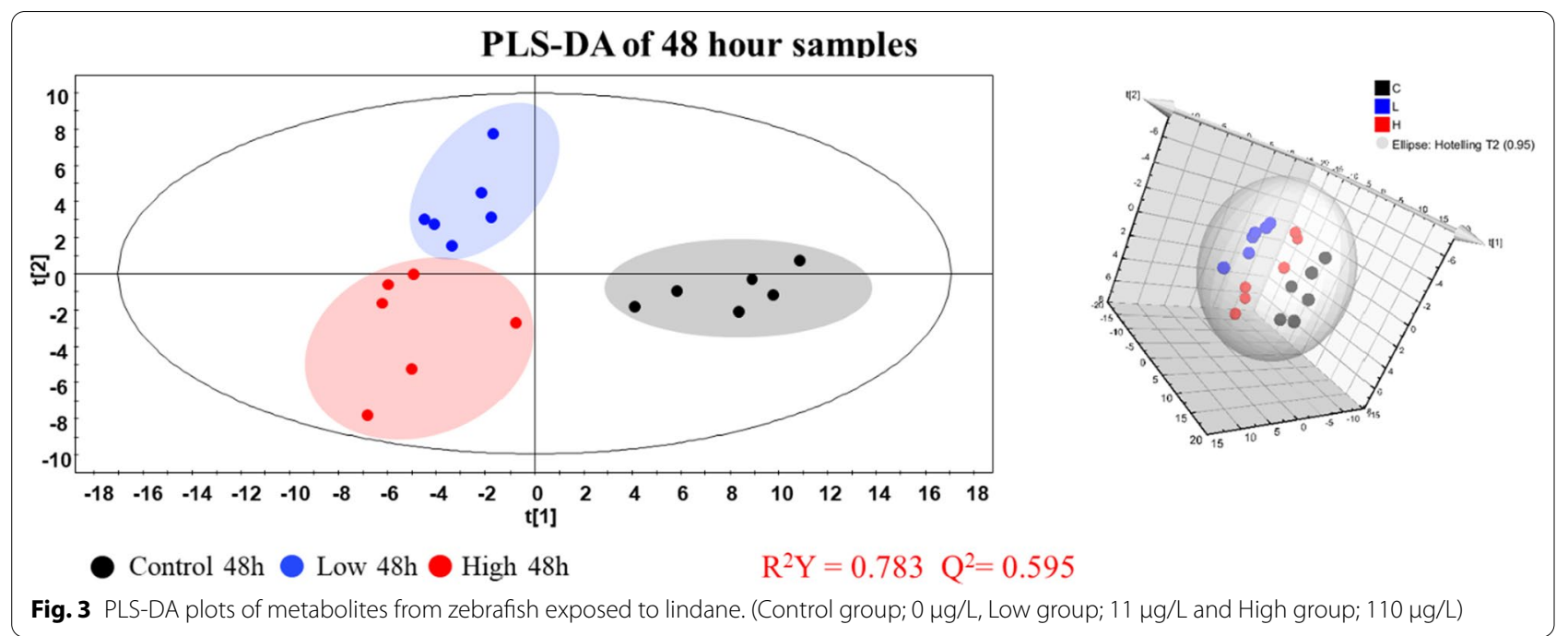

(PPP), histidine metabolism, phenylalanine metabolism, alanine/aspartate/glutamate metabolism, and phenylalanine/tyrosine/tryptophan biosynthesis (Fig. 5). The metabolic pathways that were disturbed by lindane exposure and the trends of the related metabolites are summarized in Fig. 6.

In the PPP, the levels of ribose, ribose 5-phosphate, gluconic acid, gluconic acid lactone, 6-phophogluconic acid, glyceric acid, and sedoheptulose 7-phosphate were downregulated in zebrafish after lindane exposure. The decreases in these metabolites are probably caused by downregulation of glucose-6-phosphate dehydrogenase (G6PD) gene expression after lindane exposure [45]. G6PD plays a role in the oxidative step of the PPP and catalyzes the rate-limiting step to produce NADPH; therefore, the expression and activity of the G6PD-related gene tightly regulates PPP [46]. In addition, in the non-oxidation step of PPP, erythros4-phosphate, which can contribute to the formation of aromatic amino acids such as phenylalanine, tryptophan, histidine and tyrosine, is produced, so the disturbance of PPP will affect the formation of amino acids [47].

With respect to phenylalanine/tyrosine/tryptophan biosynthesis and phenylalanine metabolism, phenylalanine and tyrosine decreased. The downregulation of these metabolites may lead to a negative impact on the synthesis of important neurotransmitters and hormones such as dopamine, epinephrine and norepinephrine [48]. Interruption of phenylalanine/tyrosine/tryptophan biosynthesis has an effect on the nervous system [19]. Moreover, an unstable phenylalanine metabolic state negatively affects the body's normal growth, development and physiological functions [49]. In a metabolomics study of zebrafish embryos that were exposed to fluoxetine, phenylalanine and tyrosine were downregulated in embryos [19].

In the present study, the relative amounts of the corresponding metabolites associated with alanine/aspartate and glutamate metabolism (alanine, glutamate and aspartate), were all downregulated. A study of metabolomics in medaka reported that alanine and tyrosine were downregulated after medaka embryos were exposed to dinoseb [50]. The downregulation of alanine and tyrosine resulted from energy expenditure $[19,50]$. In addition, alanine is an essential nitrogenous product that is produced by the intestinal catabolism of glutamine, glutamate, and aspartate [51]. Downregulation of glutamate and aspartate may negatively affect the role of alanine in transporting some dietary amino acids to intestinal tissues [52].

In histidine metabolism, the concentrations of histidine, glutamate, and aspartate were downregulated. A study that evaluated the antitumor effects on A549 cells reported that histidine plays a role in inhibiting cell proliferation and migration in lung cancer cells and has antitumor effects [53]. In addition, a metabolomics study of bovine colostrum and mature milk reported that brain and nervous system development was encouraged by histidine in newborns [54]. Therefore, a decrease in histidine may increase the risk of cancer and have devastating effects on the brain and nervous system. Moreover, glutamate, a precursor of 


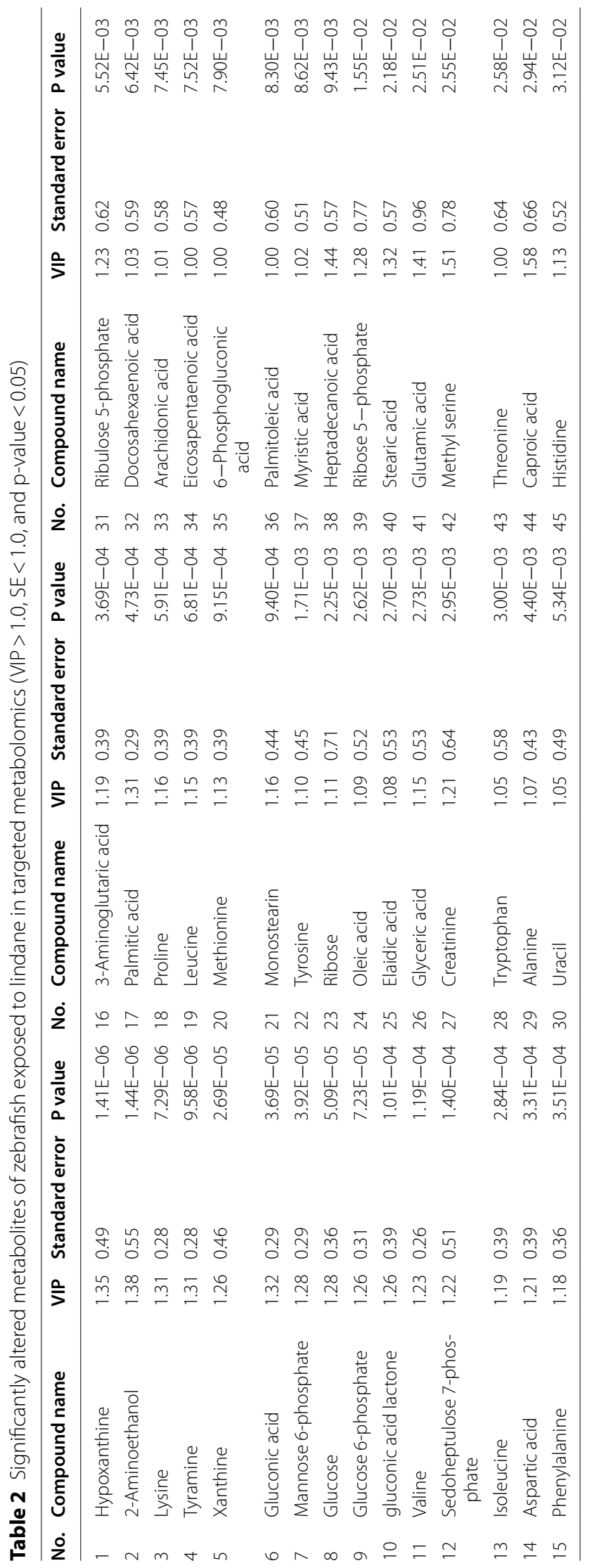




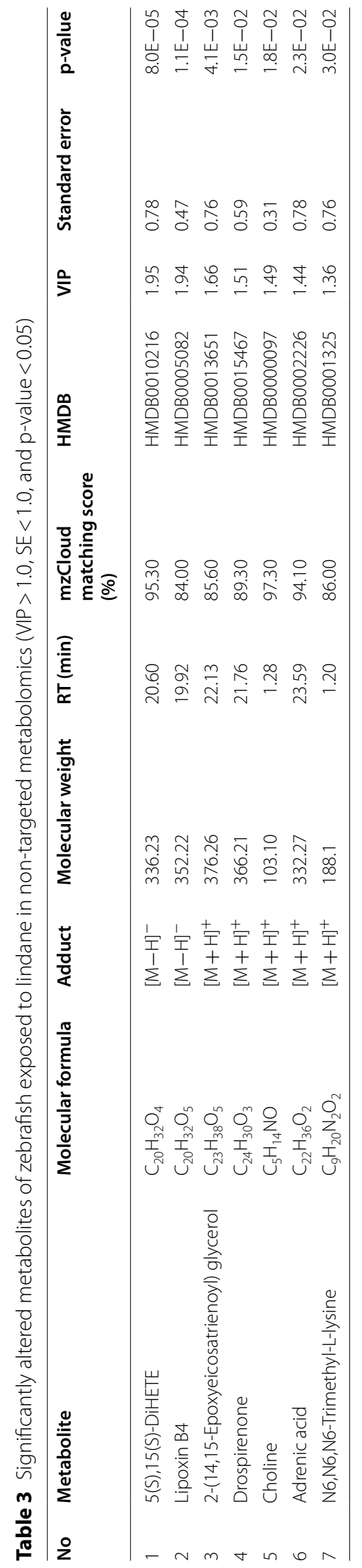




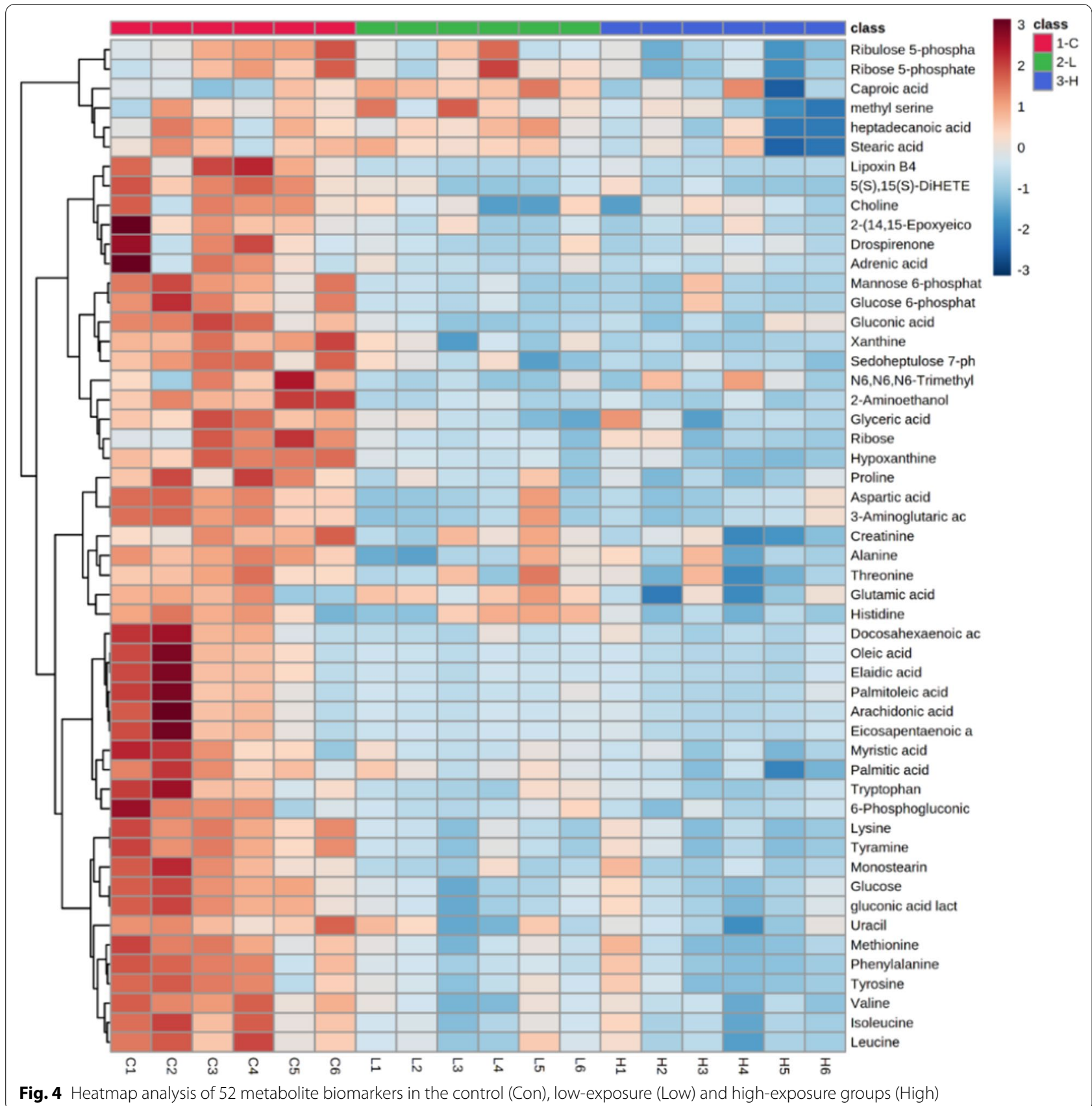

GABA, which is involved in both alanine/aspartate and glutamate metabolism and histidine metabolism was decreased in the lindane exposer groups. Lindane, a neurotoxin, is known as an inhibitor of GABA-gated chloride channel inducing partial repolarization of neurons, resulting in involuntary muscle contraction and seizures [10]. Therefore, decreasing glutamate demonstrated that neurotransmitter-mediated nervous system was unable to be regulated. 


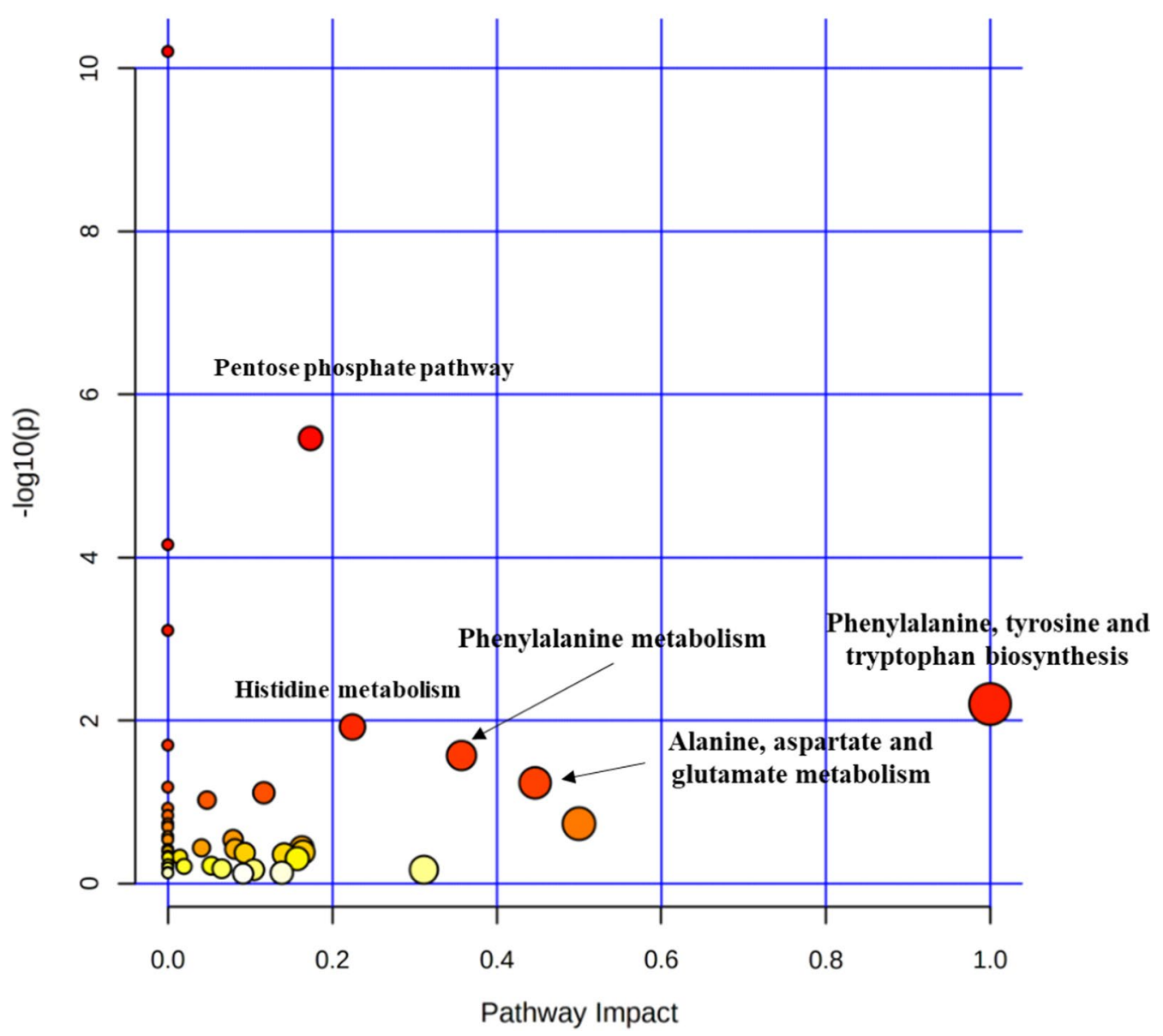

Fig. 5 Summary of pathway analyses determined by MetaboAnalyst 5.0, as visualized by bubble plots. Bubble sizes are proportional to the impact of each pathway, and bubble colors denote the degrees of significance, from highest (red) to lowest (white)

\section{Oxidative stress induced by lindane}

To confirm the oxidative stress from exposure to lindane, ROS and MDA assay tests were conducted with zebrafish. The amounts of ROS were detected by DCFH-DA assays. Excessive oxidative stress can lead to the accumulation of ROS, which will damage major cell components [55]. Higher ROS production levels may affect the oxidation of proteins and lipids, disturbance of gene expression, and disruption of cellular redox states [56]. In addition, the accumulation of ROS lead to cell apoptosis [57]. In the study of apoptosis in thyroid cancer cell, inhibition of the PPP cause ROSinduced apoptosis in thyroid cancer cells [58]. In this study, PPP was downregulated in the pathway analysis and ROS content increased, therefore, apoptosis could have been induced. MDA, one of the major products of lipid peroxidation in cell membranes, is used as an index of lipid oxidation [59]. As a result, ROS and MDA 


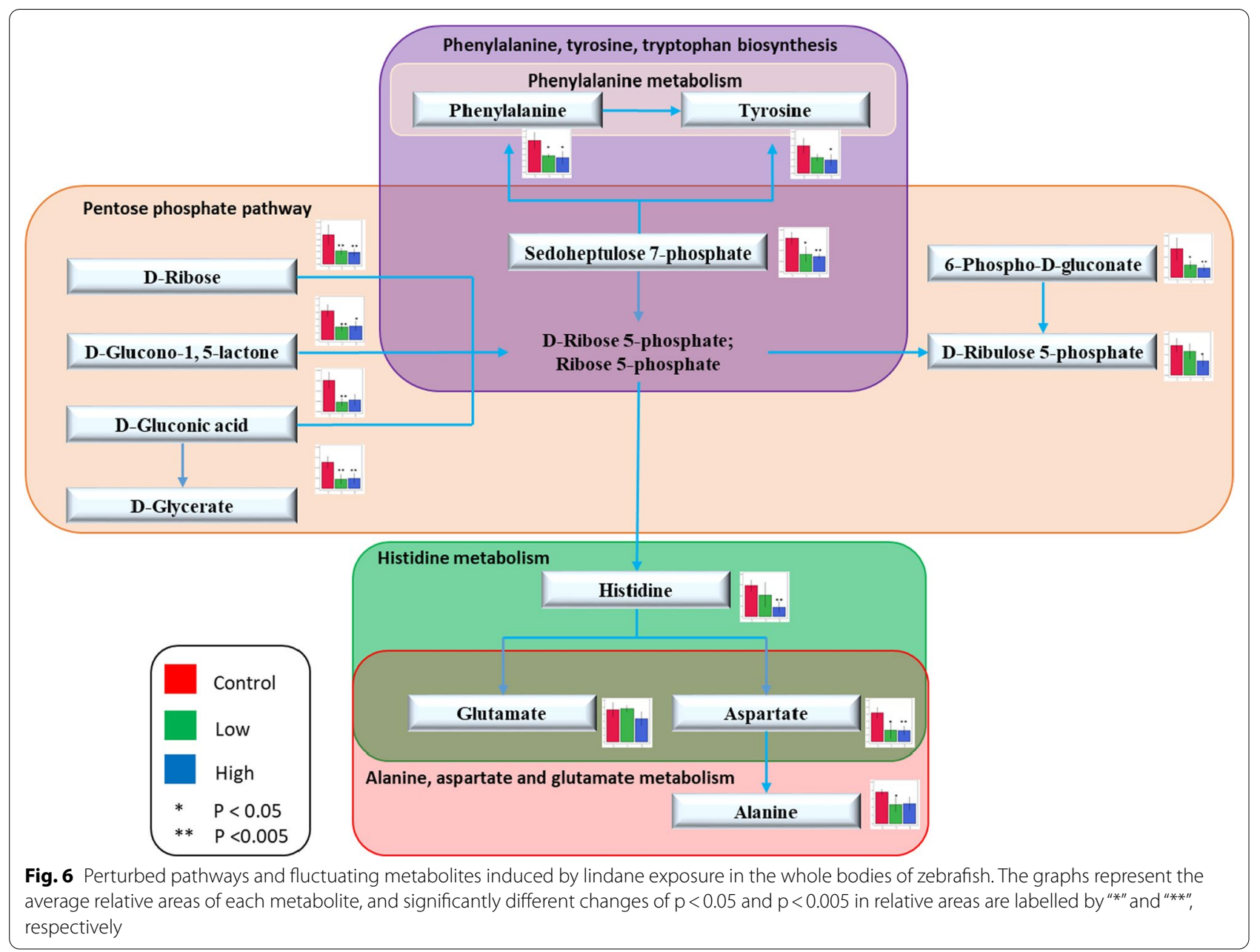

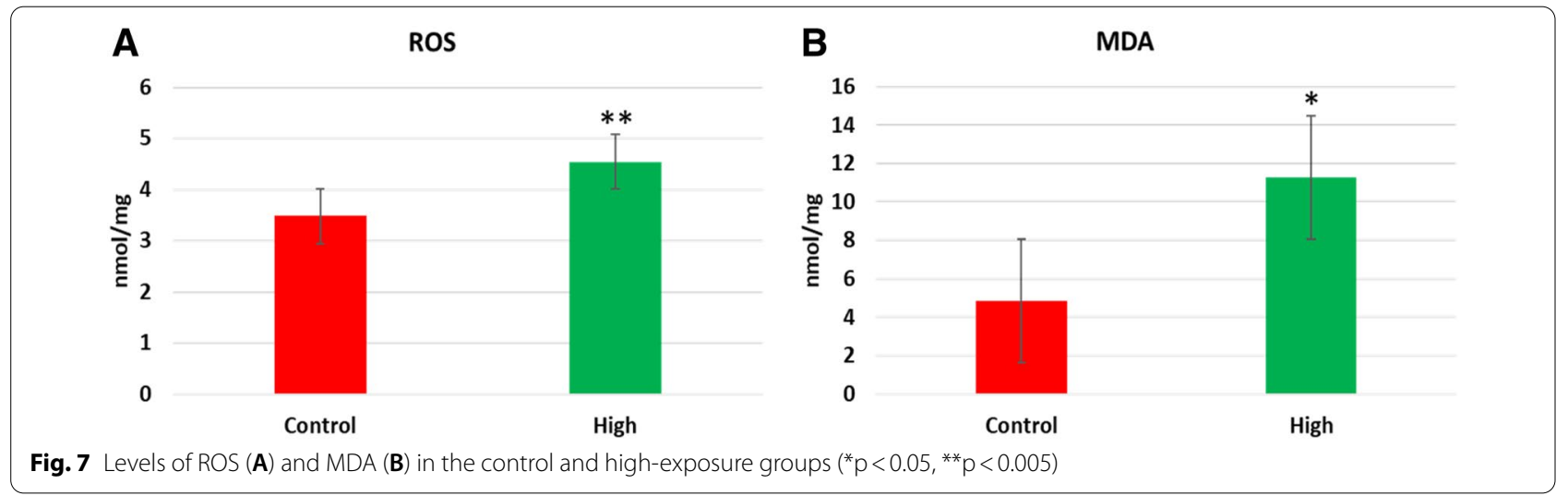

in the exposure group under high lindane concentrations were significantly increased $(\mathrm{p}<0.05)$ (Fig. 7), which suggested oxidative damage to the cells.

\section{Authors' contributions}

$\mathrm{XY}$ conducted the sample preparation and sample analysis and wrote the manuscript. JL and EP helped with the sample preparation and analysis. $\mathrm{H}-\mathrm{KL}$ revised the final manuscript. JHK supervised the project and revised the final manuscript. All authors read and approved the final manuscript. 


\section{Availability of data and materials}

All data generated or analyzed during this study are included in this published article.

\section{Declarations}

\section{Competing interests}

The authors declare that they have no competing interests.

Received: 27 May 2021 Accepted: 27 June 2021

Published online: 16 July 2021

\section{References}

1. Jones KC, De Voogt P (1999) Persistent organic pollutants (POPs): state of the science. Environ Pollut 100:209-221

2. Ashraf MA (2017) Persistent organic pollutants (POPs): a global issue, a global challenge. Springer

3. Wei D, Kameya T, Urano K (2007) Environmental management of pesticidal POPs in China: past, present and future. Environ Int 33:894-902

4. Kim J-H, Smith A (2001) Distribution of organochlorine pesticides in soils from South Korea. Chemosphere 43:137-140

5. Fu S, Chu S, Xu X (2001) Organochlorine pesticide residue in soils from Tibet, China. Bull Environ Contam Toxicol 66:171-177

6. Meijer SN, Halsall CJ, Harner T, Peters A, Ockenden W, Johnston A, Jones KC (2001) Organochlorine pesticide residues in archived UK soil. Environ Sci Technol 35:1989-1995

7. Saez J, Alvarez A, Fuentes M, Amoroso M, Benimeli C (2017) An overview on microbial degradation of lindane. Microbe-induced degradation of pesticides, pp. 191-212

8. Islam R, Lynch JW (2012) Mechanism of action of the insecticides, lindane and fipronil, on glycine receptor chloride channels. Br J Pharmacol 165:2707-2720

9. Narahashi T (2002) Nerve membrane ion channels as the target site of insecticides. Mini Rev Med Chem 2:419-432

10. Wafford K, Sattelle D, Gant D, Eldefrawi A, Eldefrawi M (1989) Noncompetitive inhibition of GABA receptors in insect and vertebrate CNS by endrin and lindane. Pestic Biochem Physiol 33:213-219

11. Copping $L$ (1999) Metabolic pathways of agrochemicals: part two-insecticides and fungicides, eds-in-chief T Roberts and D Hutson. Royal Society of Chemistry, Cambridge

12. Humphreys EH, Janssen S, Heil A, Hiatt P, Solomon G, Miller MD (2008) Outcomes of the California ban on pharmaceutical lindane: clinical and ecologic impacts. Environ Health Perspect 116:297-302

13. Misra K, Brar KS, Verma M, Tyagi R, Trivedy R, Sharma S (2007) Lindane-A contaminant of global concern. I Control Pollution 23:169-187

14. Sumith J, Parkpian P (2008) Behaviour of endosulfan and lindane during sediment elutriate and water spike toxicity tests under saline conditions. Ann Sri Lanka Dept Agric 10:231-243

15. Sandu M, Virsta A (2015) Environmental toxicity of lindane and health effect. J Environ Prot Ecol 16:933-944

16. Ezemonye LI, Ikpesu TO, Tongo I (2008) Distribution of lindane in water, sediment, and fish from the Warri River of the Niger Delta, Nigeria. Arh Hig Rada Toksikol 59:261-270

17. Dooley K, Zon LI (2000) Zebrafish: a model system for the study of human disease. Curr Opin Genet Dev 10:252-256

18. Gunnarsson L, Jauhiainen A, Kristiansson E, Nerman O, Larsson DJ (2008) Evolutionary conservation of human drug targets in organisms used for environmental risk assessments. Environ Sci Technol 42:5807-5813

19. Mishra P, Gong Z, Kelly BC (2017) Assessing biological effects of fluoxetine in developing zebrafish embryos using gas chromatography-mass spectrometry based metabolomics. Chemosphere 188:157-167

20. Song Y, Chai T, Yin Z, Zhang X, Zhang W, Qian Y, Qiu J (2018) Stereoselective effects of ibuprofen in adult zebrafish (Danio rerio) using UPLC-TOF/ MS-based metabolomics. Environ Pollut 241:730-739
21. Teng M, Zhu W, Wang D, Qi S, Wang Y, Yan J, Dong K, Zheng M, Wang C (2018) Metabolomics and transcriptomics reveal the toxicity of difenoconazole to the early life stages of zebrafish (Danio rerio). Aquat Toxicol 194:112-120

22. Elie MR, Choi J, Nkrumah-Elie YM, Gonnerman GD, Stevens JF, Tanguay RL (2015) Metabolomic analysis to define and compare the effects of PAHs and oxygenated PAHs in developing zebrafish. Environ Res 140:502-510

23. McGrath P, Li C-Q (2008) Zebrafish: a predictive model for assessing druginduced toxicity. Drug Discovery Today 13:394-401

24. Roberts LD, Souza AL, Gerszten RE, Clish CB (2012) Targeted metabolomics. Curr Protocols Mol Biol. 98, 30.32. 31-30.32. 24

25. Dettmer K, Aronov PA, Hammock BD (2007) Mass spectrometry-based metabolomics. Mass Spectrom Rev 26:51-78

26. Emwas A-H, Roy R, McKay RT, Tenori L, Saccenti E, Gowda G, Raftery D, Alahmari F, Jaremko L, Jaremko M (2019) NMR spectroscopy for metabolomics research. Metabolites 9:123

27. Yuk J, Simpson MJ, Simpson AJ (2013) 1-D and 2-D NMR-based metabolomics of earthworms exposed to endosulfan and endosulfan sulfate in soil. Environ Pollut 175:35-44

28. Wen B, Jin S-R, Chen Z-Z, Gao J-Z (2018) Physiological responses to cold stress in the gills of discus fish (Symphysodon aequifasciatus) revealed by conventional biochemical assays and GC-TOF-MS metabolomics. Sci Total Environ 640:1372-1381

29. Wishart DS (2008) Quantitative metabolomics using NMR. TrAC Trends Anal Chem 27:228-237

30. Lei Z, Huhman DV, Sumner LW (2011) Mass spectrometry strategies in metabolomics. J Biol Chem 286:25435-25442

31. Fancy S-A, Rumpel K (2008) GC-MS-based metabolomics. Biomarker methods in drug discovery and development, Springer. pp 317-340

32. Savolainen OI, Sandberg A-S, Ross AB (2016) A simultaneous metabolic profiling and quantitative multimetabolite metabolomic method for human plasma using gas-chromatography tandem mass spectrometry. J Proteome Res 15:259-265

33. Naz S, Vallejo M, García A, Barbas C (2014) Method validation strategies involved in non-targeted metabolomics. J Chromatogr A 1353:99-105

34. Castro-Puyana M, Herrero M (2013) Metabolomics approaches based on mass spectrometry for food safety, quality and traceability. TrAC Trends Anal Chem 52:74-87

35. Huang L, Zheng N, Zheng B, Wen F, Cheng J, Han R, Xu X, Li S, Wang $J$ (2014) Simultaneous determination of aflatoxin M1, ochratoxin A, zearalenone and a-zearalenol in milk by UHPLC-MS/MS. Food Chem 146:242-249

36. Ribbenstedt A, Ziarrusta H, Benskin JP (2018) Development, characterization and comparisons of targeted and non-targeted metabolomics methods. PLoS One 13:e0207082

37. Koek MM, Jellema RH, van der Greef J, Tas AC, Hankemeier T (2011) Quantitative metabolomics based on gas chromatography mass spectrometry: status and perspectives. Metabolomics 7:307-328

38. Lee H-K, Kim K, Lee J, Lee J, Lee J, Kim S, Lee S-E, Kim J-H (2020) Targeted toxicometabolomics of endosulfan sulfate in adult zebrafish (Danio rerio) using GC-MS/MS in multiple reaction monitoring mode. J Hazardous Mater 389:122056

39. Ensenbach U, Nagel R (1995) Toxicity of complex chemical mixtures: acute and long-term effects on different life stages of zebrafish (Brachydanio rerio). Ecotoxicol Environ Saf 30:151-157

40. Wen W, Zhang Z, Jiang B, Hao Y (2021) Orbitrap-MS-based untargeted metabolomics study on the therapeutic effect of colchicine on myocardial infarction. Biomed Chromatography, e5148

41. Tooby T, Durbin F (1970) 1975) Lindane residue accumulation and elimination in rainbow trout (Salmo gairdnerii Richardson) and roach (Rutilus rutilus Linnaeus. Environ Pollut 8:79-89

42. Escandón M, Meijón M, Valledor L, Pascual J, Pinto G, Cañal MJ (2018) Metabolome integrated analysis of high-temperature response in Pinus radiata. Front Plant Sci 9:485

43. t'Kindt, R., Morreel, K., Deforce, D., Boerjan, W., and Van Bocxlaer, J. (2009) Joint GC-MS and LC-MS platforms for comprehensive plant metabolomics: Repeatability and sample pre-treatment. J Chromatogr B 877:3572-3580 
44. Naz S, Moreira dos Santos DC, García A, Barbas C (2014) Analytical protocols based on LC-MS, GC-MS and CE-MS for nontargeted metabolomics of biological tissues. Bioanalysis 6:1657-1677

45. Oskarsson A, Ullerås E, Plant KE, Hinson JP, Goldfarb PS (2006) Steroidogenic gene expression in $\mathrm{H} 295 \mathrm{R}$ cells and the human adrenal gland: adrenotoxic effects of lindane in vitro. J Appl Toxicol 26:484-492

46. Patra KC, Hay N (2014) The pentose phosphate pathway and cancer. Trends Biochem Sci 39:347-354

47. Stine Z, Altman B, Hsieh A, Gouw A, Dang C (2014) Deregulation of the cellular energetics of cancer cells

48. Neurauter G, Grahmann AV, Klieber M, Zeimet A, Ledochowski M, Sperner-Unterweger B, Fuchs D (2008) Serum phenylalanine concentrations in patients with ovarian carcinoma correlate with concentrations of immune activation markers and of isoprostane-8. Cancer Lett 272:141-147

49. Wu X-H, Zhao C, Zhang A-H, Zhang J-Q, Wang X, Sun X-L, Sun Z, Wang X-J (2018) High-throughput metabolomics used to identify potential therapeutic targets of Guizhi Fuling Wan against endometriosis of cold coagulation and blood stasis. RSC Adv 8:19238-19250

50. Viant MR, Pincetich CA, Hinton DE, Tjeerdema RS (2006) Toxic actions of dinoseb in medaka (Oryzias latipes) embryos as determined by in vivo 31P NMR, HPLC-UV and 1 H NMR metabolomics. Aquat Toxicol 76:329-342

51. Wu G (1998) Intestinal mucosal amino acid catabolism. J Nutr 128:1249-1252

52. Rémésy C, Moundras C, Morand C, Demigne C (1997) Glutamine or glutamate release by the liver constitutes a major mechanism for nitrogen salvage. Am J Physiol Gastrointestinal Liver Physiol 272:G257-G264

53. Escobar-Reséndiz R, Reyes-Esparza J, Blake IO, Rodriguez-Fragoso L (2020) Evaluation of antitumoral effect of the combination of I-histidine methyl esther hydrochloride of Anfotericin b with antineoplastics on A549 cells. FASEB J 34:1-1

54. Li M, Li W, Kong F, Kang S, Liang X, Han H, Wu J, Zheng Y, Li Q, Yue X (2020) Metabolomics methods to analyze full spectrum of amino acids in different domains of bovine colostrum and mature milk. Eur Food Res Technol 246:213-224

55. Feng L, Wu H-W, Song G-Q, Lu C, Li Y-H, Qu L-N, Chen S-G, Liu X-M, Chang Q (2016) Chronical sleep interruption-induced cognitive decline assessed by a metabolomics method. Behav Brain Res 302:60-68

56. Kovacik A (2017) Oxidative stress in fish induced by environmental pollutants. Sci Papers Anim Sci Biotech 50:121-125

57. Mele L, Paino F, Papaccio F, Regad T, Boocock D, Stiuso P, Lombardi A, Liccardo D, Aquino G, Barbieri A (2018) A new inhibitor of glucose6-phosphate dehydrogenase blocks pentose phosphate pathway and suppresses malignant proliferation and metastasis in vivo. Cell Death Dis 9:1-12

58. Liu C-L, Hsu Y-C, Lee J-J, Chen M-J, Lin C-H, Huang S-Y, Cheng S-P (2020) Targeting the pentose phosphate pathway increases reactive oxygen species and induces apoptosis in thyroid cancer cells. Mol Cell Endocrinol 499:110595

59. Mateos R, Lecumberri E, Ramos S, Goya L, Bravo L (2005) Determination of malondialdehyde (MDA) by high-performance liquid chromatography in serum and liver as a biomarker for oxidative stress: application to a rat model for hypercholesterolemia and evaluation of the effect of diets rich in phenolic antioxidants from fruits. J Chromatogr B 827:76-82

\section{Publisher's Note}

Springer Nature remains neutral with regard to jurisdictional claims in published maps and institutional affiliations.

\section{Submit your manuscript to a SpringerOpen ${ }^{\circ}$ journal and benefit from:}

- Convenient online submission

- Rigorous peer review

- Open access: articles freely available online

- High visibility within the field

- Retaining the copyright to your article

Submit your next manuscript at $\boldsymbol{\nabla}$ springeropen.com 